\section{A case report of a pulmonary abscess}

\section{Case presentation}

A 34 year-old male, with history of lung ALK positive adenocarcinoma (subdue to lobectomy and treated with adjuvant chemotherapy and radiotherapy), that posteriorly developed distal esophagus adenocarcinoma (immunohistochemically different from the lung), affecting the cardia. Was surgically proposed to eso-gastrectomy and eso-gastrostomy. Due the partial dehiscence of the anastomosis, it was placed an endoluminal prosthesis. One month after, during the removal of the prosthesis, that wasn't possible, a second one was placed (stent in stent). Three weeks later, in the attempt of removing the inner prosthesis, it was found an esophageal orifice, probably of iatrogenic cause. A chest-computerized axial tomography revealed (images 1,2,3,4 and 5) multiple esophageal fistulas and one that was inducing a pulmonary abscess of $16 \mathrm{~cm}$. He was started on empiric antibiotherapy with metronidazole, clindamycin and fluconazole. A new esophageal prosthesis was placed and percutaneous drainage was performed, and its microbiological study revealed: klebsiella pneumoniae carbapenemase (KPC), with CIM for meropenem of 16 and a Candida cruzei. The anthiobiogram showed: CIM Meropenem: 16 mg/L; CIM Ertapenem: 16 mg/L; CIM Colystin: $1 \mathrm{mg} / \mathrm{L}$ and CIM Tigecycline: 0,38 mg/L. Accordingly, to the antibiogram, was started on tigecycline, colistine and amphotericin B. However, there wasn't any improvement, and the patient died on $45^{\text {th }}$ day of his admission.

\section{Discussion}

The emergency of multirresistent microorganisms it's a challenge for treatment, control and prevention. ${ }^{3}$ When these infections involve abscesses, adequate drainage and an appropriate long term antibiotic therapy has shown an improvement in the outcome. ${ }^{3}$ However, there is some debate concerning the appropriate dosage and the most favorable pharmacokinetic/pharmacodynamic profiles in this cases. Unfortunately the optimal treatment is unknown. The use of aminoglycosides, polymyxin combinations and tigecycline appeared to have higher success rates. ${ }^{1,2}$

Obviously, in this case, due to its clinical past history and immunosuppression, even the best approach wasn't enough.

\section{References}

1. Narayanan N; Jonhson L; MacDougall C. Beyond susceptible and Resistant, Part III: Treatment of infections due to Gram-Negative

2. Organisms producing carbapenemases. J Pediatr Pharmacol Ther. 2016. 21(2): 110-119.

3. Morrill HJ, Pogue JM, Kaye KS, LaPlante KL. Treatment options for carbapenem-resistant enterobacteriaceae Infections. Open Forum Infect Dis. 2015; 2(2): 1-11

4. Di Carlo P, Pantuso G, Cusimano A, et al. Two cases of monomicrobial intraabdominal abscesses due to KPC - 3 Klebsiella pneumoniae ST258 clone. BMC Gastroenterology. 2011;11:103.

\section{Diagnóstico: Pulmonary abscess due to KPC-3 Klebsiella pneumoniae}

\section{Tatiana Fonseca ${ }^{1}$, Maria Ana Canelas ${ }^{1}$}

${ }^{1}$ Department of Internal Medicine, Centro Hospitalar Vila Nova de Gaia Espinho, Vila Nova de Gaia, Portugal

Correspondencia: tatifonseca_84@hotmail.com

Como citar este artículo: Fonseca T, Canelas MA

Pulmonary abscess due to KPC-3 Klebsiella pneumoniae. Galicia Clin 2017; 78 (1): XX-XX

Recibido: 08/07/2016; Aceptado: 18/07/2016
Images 1-5: Multiple esophageal fistulas (black arrow) and one that was inducing a pulmonary abscess of $16 \mathrm{~cm}$ (white arrow)
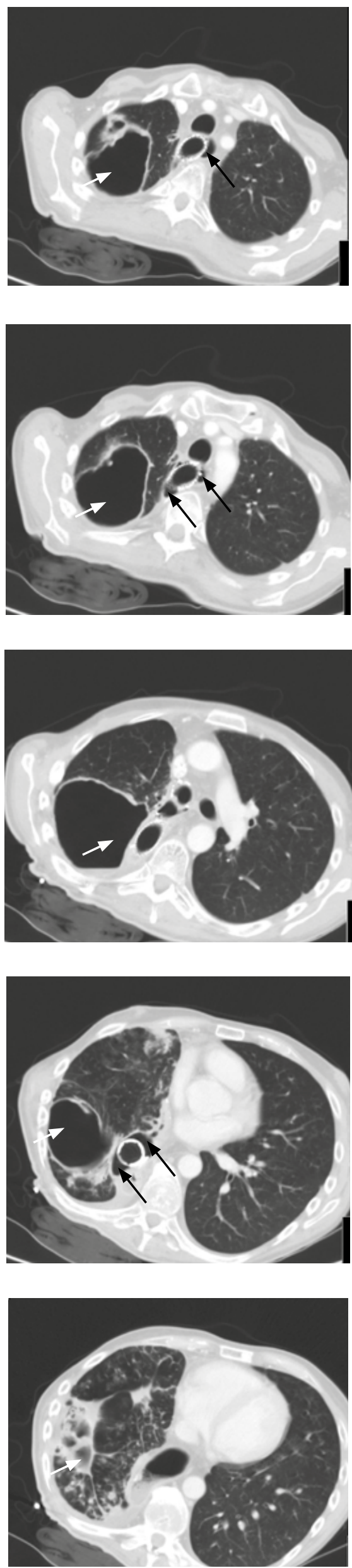